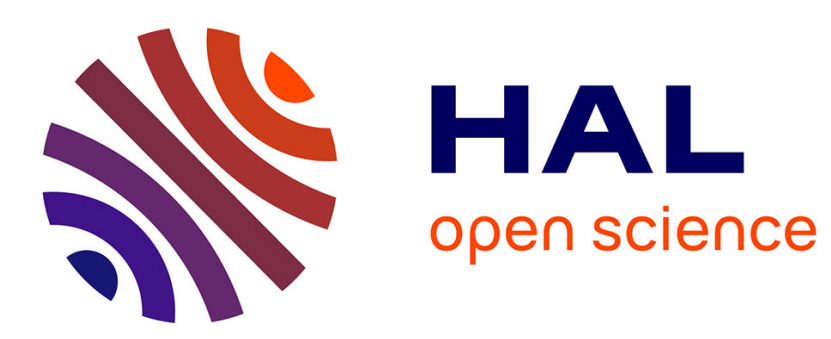

\title{
Contenu et effets de la proximité institutionnelle : un cas d'enfermement dans l'industrie aéronautique
}

\author{
Ibtissem Hamouda, Damien Talbot
}

\section{To cite this version:}

Ibtissem Hamouda, Damien Talbot. Contenu et effets de la proximité institutionnelle : un cas d'enfermement dans l'industrie aéronautique. Revue management \& avenir, 2018, 101 (3), pp.105. 10.3917/mav.101.0105 . hal-02329612

\section{HAL Id: hal-02329612 \\ https://hal.science/hal-02329612}

Submitted on 23 Oct 2019

HAL is a multi-disciplinary open access archive for the deposit and dissemination of scientific research documents, whether they are published or not. The documents may come from teaching and research institutions in France or abroad, or from public or private research centers.
L'archive ouverte pluridisciplinaire HAL, est destinée au dépôt et à la diffusion de documents scientifiques de niveau recherche, publiés ou non, émanant des établissements d'enseignement et de recherche français ou étrangers, des laboratoires publics ou privés. 


\title{
Contenu et effets de la proximité institutionnelle : un cas d'enfermement dans l'industrie aéronautique ${ }^{1}$ \\ Components and effects of institutional proximity: a lock in evidence in the aeronautical industry
}

\author{
Ibtissem Hamouda, Doctorante en Sciences de Gestion \\ ibtissem.hamouda@uca.fr \\ Damien Talbot, Professeur en Sciences de Gestion \\ damien.talbot@uca.fr \\ CRCGM - Université Clermont Auvergne \\ 49 Boulevard François Mitterrand - 63000 Clermont-Ferrand
}

Paru dans Management et Avenir, 2018, vol. 3, n¹01, pp. 105-129

\section{Résumé}

Si divers travaux se sont attachés à mesurer et caractériser certaines dimensions de la proximité, la proximité institutionnelle reste probablement la moins étudiée : cet article vise à combler ce manque. Nous proposons d'analyser la proximité institutionnelle par l'étude des représentations collectives portées par les acteurs en réalisant une analyse de contenu thématique. Dans une visée compréhensive, 11 acteurs de l'aéronautique ont été interrogés sur leur perception de cette proximité. L'analyse des discours nous a permis de mettre au jour le contenu de la proximité institutionnelle et ses effets sur les relations interorganisationnelles. En particulier, une perception positive mais aussi négative de l'industrie aéronautique apparaît, ce qui est une nouveauté, tandis qu'un effet inattendu d'enfermement (lock in) se fait jour.

Mots-clés : proximité institutionnelle - représentations partagées - sous-traitance - industrie aéronautique.

\begin{abstract}
Research on the dimensions of proximity draws on the measurement and the characteristics of these dimensions of proximity though the institutional proximity lacks clarity. This paper tries to fill this gap. Indeed, we propose to analyze the institutional proximity by the observation of shared representations. To meet this target, we realize a thematic content analysis. To provide a comprehensive view, we interviewed 11 aeronautical actors on their perception of this proximity. Through the analysis of their verbatims, the research highlighted the components and the effects of institutional proximity on their inter-organizational relations. The study reveals a positive and a negative shared perception of the sector which is a new finding despite an unexpected effect of lock in came under light.
\end{abstract}

Key words: institutional proximity - shared representations - subcontracting - aeronautical industry.

\footnotetext{
${ }^{1}$ Une première version de ce papier a été présentée lors du 10ème colloque GeCSO qui s'est tenu à Montréal en juin 2017.
} 


\section{INTRODUCTION}

L'école de la proximité s'est développée en France depuis une vingtaine d'années grâce à des auteurs qui font de l'espace un objet central d'analyse. D'emblée, l'espace est compris d'un point de vue géographique et social (Bellet et al., 1993) : cette école se construit autour du principe fondateur selon lequel la localisation dans un espace géographique et social conditionne les interactions entre acteurs (Gilly et Torre, 2000 ; Pecqueur et Zimmermann, 2004 ; Boschma, 2005 ; Bouba-Olga et Grossetti, 2008 ; Carrincazeaux et al. 2008 ; Rychen et Zimmermann, 2008). Rapidement, et pour que la dimension géographique ne reste pas « un impensé de la gestion » (Lauriol et al, 2008, p. 92), de nombreux auteurs en Sciences de Gestion se sont emparés de ces questions, comme Adam-Ledunois et Renault (2008), Loilier (2010), Angué et Mayrhofer (2010), Gomez et al. (2011), Talbot (2013) ou encore Pihel et Journé (2016).

Pour tous, la proximité présente plusieurs dimensions. La proximité géographique réunit des acteurs co-localisés qui simultanément développent des proximités non géographiques. Le nombre de dimensions varie selon les auteurs. Torre et Rallet (2005) définissent la proximité organisée comme la capacité qu'offre une organisation de faire interagir ses membres. Boschma (2005) distingue les proximités cognitive (partage de connaissances), sociale (appartenance à un réseau social), organisationnelle (appartenance à une organisation) et institutionnelle (partage d'institutions formelles et informelles).

L'évaluation de ces proximités s'avère être un travail essentiel car leurs effets sur les interactions sont fonction de leur intensité. Par exemple une proximité géographique trop forte peut induire des effets de congestion spatiale, une proximité cognitive trop faible peut conduire à une situation d'incompréhension compromettant les performances innovatrices des entreprises en relation (Broekel et Boschma, 2012 ; Boschma, 2005) ou encore une proximité institutionnelle élevée peut générer une situation d'enfermement (Boschma, 2005; Detchenique et Loilier, 2016). Certaines dimensions ont déjà fait l'objet d'un travail de caractérisation et d'évaluation. Ainsi, la proximité géographique peut être identifiée par la distance spatiale qui sépare deux acteurs (Gilly et Torre, 2000, Pecqueur et Zimmermann, 2004) : concrètement, deux acteurs sont d'autant plus proches géographiquement qu'ils sont situés dans le même NUTS ${ }^{2}$ (Rychen et Zimmermann, 2008). L'observation des brevets déposés par les acteurs est une façon d'évaluer la proximité cognitive : la comparaison en termes de similarité des classes de brevets peut mettre à jour des similarités dans les bases de connaissances (Breschi et al. 2003; Bennera et Waldfogelb, 2008 ; Hautala, 2011). La proximité sociale est classiquement évaluée par les outils d'analyse de réseaux (Lazega, 1994) et plus précisément par la distance géodésique (c'est-à-dire la plus courte) qui sépare deux acteurs (Boschma et Frenken, 2009 ; Balland, 2012), tandis que la proximité organisationnelle l'est par le fait d'appartenir ou non à une même organisation (Torre et Rallet, 2005). Dans ce dernier cas, s'ajoutent les travaux sur les configurations organisationnelles (Fiss, 2007) qui conduisent à proposer des typologies qui rassemblent des organisations similaires : cet indicateur porte sur des similitudes de caractère, par exemple la forme juridique et la taille.

En revanche, du fait de sa nature (le partage des lois, des règles, des coutumes, des représentations et plus généralement des institutions), la proximité institutionnelle est plus difficile à révéler (Kirat et Lung, 1999). Dès lors peu ou pas de travaux se sont attachés à montrer ce qu'est la proximité institutionnelle et quel est son impact dans les relations interorganisationnelles. Cet article vise à combler ce manque. Nous montrons en effet que la proximité institutionnelle peut être définie comme un ensemble de règles et de représentations partagées qui rend les attitudes des acteurs qui la partagent à la fois plus similaires et plus

\footnotetext{
${ }^{2}$ La Nomenclature des Unités Territoriales Statistiques est une classification européenne visant à faciliter les comparaisons entre pays, ou entre régions, d'un même ensemble.
} 
complémentaires (Talbot, 2008). Si cette convergence des attitudes autorise l'action collective (effet positif de la proximité institutionnelle), elle peut aussi conduire à une situation d'enfermement (effet négatif) (Grabher, 1993). C'est précisément le lien entre proximité institutionnelle, attitudes des acteurs et enfermement que cet article explore. Notre problématique est la suivante : comment la proximité institutionnelle influe-t-elle sur les attitudes des acteurs jusqu'à pouvoir créer une situation d'enfermement?

Pour ce faire, nous utilisons une méthodologie permettant d'accéder à la proximité institutionnelle basée sur l'analyse du discours des acteurs visant à rendre compte des représentations partagées et d'attitudes récurrentes. Sur le plan empirique, nous avons choisi le secteur aéronautique car il s'agit d'une industrie de souveraineté qui se caractérise par de fortes valeurs communes liées à son histoire, à son caractère stratégique pour les Etats et à la spécificité du produit avion nécessitant d'allier sécurité et haute technologie. Par ailleurs, en matière aéronautique, la sous-traitance est pratiquée de longue date (Bécue et al., 2014). Et parce qu'elle est devenue très vite internationale, la nécessité de normes communes pour le développement, la fabrication et la maintenance des aéronefs s'est imposée.

Ce travail exploratoire s'organise de la façon suivante: dans une première partie nous définissons la proximité institutionnelle et nous soulignons ses effets. Puis nous présentons la méthode suivie pour analyser la proximité institutionnelle dans une deuxième partie. Nous qualifions les représentations partagées grâce à la mise au jour de thèmes récurrents ainsi qu'un ordonnancement de ce contenu en utilisant la "fréquence d'apparition ». Nous présentons les résultats dans une troisième partie et nous discutons dans un quatrième temps du contenu et des effets. Nous proposons en dernier lieu une conclusion et des perspectives de recherche.

\section{LA PROXIMITE INSTITUTIONNELLE : DEFINITION ET EFFETS}

Nous revenons dans cette partie sur les différentes définitions de la proximité institutionnelle, puis déterminons deux catégories d'effets de cette proximité sur les comportements.

\subsection{DEFINITION : REGLES ET REPRESENTATIONS PARTAGEES}

La proximité institutionnelle se définit dans ce travail comme un partage d'institutions, c'està-dire de lois, de règles, de coutumes, de valeurs, de représentations etc. (Talbot, 2008) (cf. tableau 1 pour une synthèse des travaux les plus significatifs et les plus usités). Selon North (1994), les institutions sont d'abord des contraintes qui, fournissant la structure d'incitations du jeu économique, définissent et limitent les choix des individus. Elles se subdivisent en contraintes formelles ou informelles : dans le premier cas, ce sont les règles que les humains conçoivent à l'exemple des contrats, dans le second cas ce sont des conventions ou des codes de comportements qui permettent de ne pas repenser systématiquement les termes de l'échange quotidien.

La proximité institutionnelle est étudiée selon les auteurs à deux niveaux d'analyse différents et complémentaires: au niveau des pays et des régions (niveau macro) et au niveau organisationnel (niveau méso) (Knoben et Oerlemans, 2006).

Au niveau macro, la proximité institutionnelle est associée au cadre politique et institutionnel qui encadre les interactions. Elle constitue un arrangement institutionnel global (Aoki, 2006). Elle recouvre à la fois des « règles du jeu » (cadre juridique, règles) (Amable et Palombarini, 2005) et l'idée d'une culture commune (langue, façons de voir le monde, représentations communes, systèmes de croyances, cartes mentales, catégorisations et classifications) (Hofstede, 1993 ; Scott, 1995 ; Adam-Ledunois et al., 2010 ; Phanuel et Renault, 2015). 
Au niveau meso, la proximité institutionnelle est comprise comme le fait d'appartenir à une même forme institutionnelle (Balland, 2012). Plusieurs formes peuvent être distinguées : ce peut être une industrie, une université ou une institution publique comme un gouvernement, chacune suivant des règles qui lui sont propres. Les institutions prennent ici la forme de règles contractuelles régissant les comportements entre et dans les organisations. Sur le plan informel, la proximité institutionnelle renvoie à la notion de culture inter-organisationnelle (Lu et al., 2016), dont la convergence est un élément-clé pour faciliter les relations entre les firmes d'un même secteur (Phillips, 1994 ; Klimas et Twarog, 2015 ; Wu et Pullman, 2015 ; Klimas, 2016). Par exemple, dans le cadre de commandes industrielles, les donneurs d'ordres en transmettant les cahiers des charges matérialisent et transportent des catégorisations (Carton et Farastier 2012).

Au-delà de ces aspects culturels, les institutions informelles prennent la forme de représentations publiques et sociales (Kirat et Lung, 1999). Ces représentations communes ne sont pas identiques par coïncidence, mais sont inculquées aux acteurs afin que tous observent des conduites coordonnées et intelligibles du point de vue du groupe (Descombes, 1996). La proximité institutionnelle relève dans ce cas du partage des représentations entre des organisations (Colletis, 2010). Ce concept de représentations collectives (Durkheim, 1898) ou sociales (Moscovici, 1976) ${ }^{3}$ est à l'origine issue des travaux en psychologie sociale visant à expliquer le modèle de construction du monde social et de la culture (Jodelet, 2003 ; Shaw, 2015). Les représentations comme des systèmes d'interprétation de l'environnement orientent ici et organisent les conduites et les communications sociales (Abric, 1994 ; Michel, 1999 ; Jodelet, 2003 ; Gardes et al., 2013). Ainsi, les représentations influencent les façons de comprendre et de traiter une tâche ainsi que le comportement des membres d'une même organisation (Laroche, 1995).

\section{Tableau 1 : Définitions de la proximité institutionnelle.}

\begin{tabular}{|c|c|}
\hline Eléments de définition & Auteurs \\
\hline $\begin{array}{l}\text { Elle se définit comme le partage d'un même environnement économique et social par les } \\
\text { acteurs en relation comprenant d'une part une dimension formelle (lois, règlementation en } \\
\text { vigueur, normes) et d'autre part une dimension informelle (habitudes, culture). } \\
\text { L'environnement institutionnel influence alors les interactions, les encourage et les contraint. }\end{array}$ & $\begin{array}{l}\text { Boschma, } \\
2005 .\end{array}$ \\
\hline $\begin{array}{l}\text { La proximité institutionnelle " concerne le partage de diverses institutions plus ou moins } \\
\text { formelles comme des lois, des règles, des coutumes, des valeurs, etc. » (Kirat et Lung, 1995. p. } \\
\text { 6). } \\
\text { Les institutions fournissent un cadre stable aux relations inter- organisationnelles. Sur le plan } \\
\text { informel, elles prennent la forme de représentations publiques et sociales. }\end{array}$ & $\begin{array}{l}\text { Kirat et Lung, } \\
1995 \text {; } \\
\text { Kirat et Lung, } \\
1999 .\end{array}$ \\
\hline $\begin{array}{l}\text { "(...) la proximité institutionnelle [ne se réduit pas] à une seule dimension cognitive } \\
\text { renvoyant à une vision partagée du monde ; nous ajoutons à la définition de la proximité } \\
\text { institutionnelle une dimension politique régulatrice qui attribue des rôles à des acteurs } \\
\text { hétérogènes afin d'apaiser transitoirement les conflits. " (p. 302). }\end{array}$ & Talbot, 2008. \\
\hline $\begin{array}{l}\text { Sur la base de cette proximité institutionnelle, les relations complexes deviennent possibles } \\
\text { entre des acteurs. C'est le cas des coutumes, des habitudes collectives, des préjugés, des règles } \\
\text { morales ou légales mais aussi des autorités, des entreprises, des associations, etc., lesquelles } \\
\text { constituent concrètement des manifestations de l'institution (notre traduction). }\end{array}$ & $\begin{array}{l}\text { Kechidi et } \\
\text { Talbot, } 2010 .\end{array}$ \\
\hline
\end{tabular}

\subsection{EFFETS DE LA PROXIMITE INSTITUTIONNELLE : SIMILARITE ET COMPLEMENTARITE DES COMPORTEMENTS}

\footnotetext{
${ }^{3}$ Pour une plus grande clarté de nos propos, nous utilisons à partir de ce point le mot «partagées ». Les qualificatifs «collectives » ou «sociales » renvoient au même sens mais sont connotés à leurs disciplines et auteurs originels.
} 
Lorsque des individus et des organisations partagent les mêmes institutions formelles et informelles, ils sont plus enclin à interagir et coordonner leurs comportements (Boschma, 2005 ; Ponds et al., 2007). Deux effets, de similarité et de complémentarité, sont à l'œuvre.

Le premier de ces effets renvoie au fait que chaque institution établit des modèles de penser et de faire : ainsi, elles rendent ces derniers plus semblables (Hodgson, 2002) en contraignant les actions dans une logique instrumentale avec un système de récompenses et de punitions (Scott, 1995). DiMaggio et Powell (1983) affirment que si les organisations partagent des informations et développent des comportements similaires c'est parce qu'elles évoluent dans un même environnement institutionnel. Cet environnement possède alors des caractéristiques particulières pouvant expliquer la similarité de comportements des organisations en concurrence au sein d'un même secteur (Hislop, 2000 ; Geels, 2014 ; Wu et Pullman, 2015 ; Martinez-Ferrero et Garcia-Sánchez, 2016), particulièrement lorsque l'incertitude y est forte (Hamadi et al., 2014). Parfois, cette similarité pose problème. D'après Moati (2013) et Boschma (2005), les représentations partagées au sein d'un secteur peuvent être une source d'inertie des comportements et un frein à l'adaptation du secteur aux transformations de son environnement. Ainsi, l'inertie institutionnelle peut conduire à un enfermement (lock in) (Grabher, 1993) empêchant tout changement, les acteurs en position dominante cherchant à maintenir l'ordre en place puisque la situation institutionnelle satisfait leurs intérêts (Amable et Palombarini, 2005). Une institution attribue un pouvoir politique à certains agents et contribue ainsi à maintenir un statu quo (Aoki, 2006). Dans ce cas, la proximité institutionnelle joue un rôle négatif en empêchant l'émergence de nouveaux comportements, partant du principe que trop de similarité freine la nouveauté. Nous proposons une revue de la littérature des éléments de définition de l'enfermement dans le tableau 2.

Tableau 2 : Définitions de l'enfermement (lock in).

\begin{tabular}{|c|c|}
\hline Eléments de définition & Auteurs \\
\hline $\begin{array}{l}\text { L'enfermement signifie un manque d'ouverture et de flexibilité. Il réduit le champ } \\
\text { des possibles. }\end{array}$ & Boschma, 2005. \\
\hline Il équivaut à une situation d'irréversibilité. & $\begin{array}{l}\text { Boschma, } 2005 . \\
\text { Mazaud, } 2006 .\end{array}$ \\
\hline Il équivaut à une situation de dépendance. & $\begin{array}{l}\text { Suh et Houston, } 2010 \\
\text {; Schmitz, Schweiger } \\
\text { et Daft, } 2016 .\end{array}$ \\
\hline $\begin{array}{l}\text { Il renvoie à l'idée de verrouillage en raison de règles discriminantes : exclusion des } \\
\text { nouveaux entrants potentiels dans le groupe social. Les membres maintiennent alors à } \\
\text { l'extérieur les acteurs susceptibles de ne pas appliquer leurs propres règles ou } \\
\text { susceptibles de ne pas partager les valeurs du groupe. }\end{array}$ & $\begin{array}{l}\text { Arthur, } 1989 \text {; } \\
\text { Leroux, Pujol et } \\
\text { Rigamonti, } 2012 .\end{array}$ \\
\hline
\end{tabular}

Toutefois, la similarité n'est jamais totale. Le second effet est relatif au partage d'institutions formelles et informelles qui va influencer la façon dont les actions sont coordonnées (Kirat et Lung 1999) et structurer les comportements (Hodgson, 2006). En effet, cette proximité permet à chacun de se voir attribuer un rôle. Les institutions ne mettent pas en présence deux acteurs sans liens, mais « deux partenaires qui doivent faire des choses différentes et dont les rôles et les statuts sont justement fixés par une règle établie, un usage social que les gens suivent » (Descombes, 1996, p. 297). En d'autres termes chacun " tient son rôle ", ce qui consiste à répondre ainsi aux attentes des autres membres ou de partenaires extérieurs sans transgresser les règles (Lagroye et al., 2006). Cette prise de rôle peut supposer une interprétation de la part de l'acteur qui le construit en fonction de la situation (Rojot, 2005). Au final, cette complémentarité autorise l'action collective.

\section{DONNEES ET METHODE}


En raison de la vocation compréhensive du projet de recherche, l'étude empirique réalisée est de nature exploratoire et la méthode d'investigation retenue est qualitative. Nous proposons une étude de cas unique compte tenu de la rareté du phénomène étudié (Yin, 1990) afin de se rapprocher du contexte des acteurs (David, 2003). Pour donner un contenu à la proximité institutionnelle et analyser ses effets, nous utilisons des données qualitatives, ici le discours. Nous identifions alors que les éléments informels de la proximité institutionnelle, ce type de données ne donnant pas accès aux éléments formels (règles formalisées, contrats, etc.).

\subsection{DONNEES : LE DISCOURS}

Les termes de « culture similaire » (Marrocu et al., 2013), de " cartes cognitives communes » (Torre, 2014), de «représentations communes» (Gilly et Lung, 2005 ; Bouba-Olga et Grossetti, 2008 ; Balland, 2012 ; Molina-Morales et al., 2015 ; Emin et Sagot-Duvauroux, 2016) ou «collectives » (Dupuy et Torre, 2004) ou de "systèmes de représentations et de croyances » (Bouba-Olga et Grossetti, 2008) sont mentionnés comme des outils de mesure possibles à la proximité institutionnelle. Pour autant, ceux-ci ne sont pas toujours explicitement définis et encore moins utilisés empiriquement. Nous nous inscrivons dans les pistes ainsi esquissées en évaluant la proximité institutionnelle par l'étude des représentations collectives qui se traduisent par des attitudes et des comportements (Ehlinger, 1998). Les travaux de Berger et Luckmann (1966) dans le champ de la psychologie cognitive ont en effet montré que les interactions interindividuelles dans un contexte social spécifique permettent de construire ensemble une connaissance sociale commune qui influence à son tour les attitudes et in fine les actions effectives des acteurs en relation.

Finalement, la question devient : comment rendre observable des représentations partagées ? Moscovici (1976) met en évidence qu'une représentation partagée est constituée autour d'un noyau figuratif qui regroupe les représentations centrales. Cette dernière notion, reprise et élargie par Abric (1994), met en avant l'organisation de la représentation sociale où les éléments centraux sont ceux qui sont décisifs pour la signification de l'objet représenté. Dans cette approche, la représentation sociale est définie comme "une structure hiérarchisée d'éléments cognitifs »(Gardes et al., 2013, p. 161). Son étude constitue une contribution décisive car elle montre qu'il est possible de rendre compte de ces représentations au moyen de méthodologies qualitatives basées sur le discours des acteurs (Negura, 2006). En effet, le discours est un support des contenus représentatifs d'un individu permettant l'étude du phénomène cognitif (Jodelet, 2003). Le discours devient alors la clé d'entrée au contenu des représentations partagées.

\subsection{L'ECHANTILLON}

Les entretiens ont été administrés dans le cadre d'un travail doctoral plus large financé par la Région Auvergne-Rhône-Alpes et qui visent à traiter les diverses dimensions de la proximité selon le cadre de Boschma (2005). Nous avons orienté les répondants vers la politique de normalisation du secteur et la mise en place de celle-ci au sein de leur organisation (éléments objectifs) ainsi que leur ressenti vis-à-vis de celle-ci (éléments subjectifs). Nous avons interrogé 9 cadres d'entreprises spécialisées dans la production en petite et moyenne série (cas 1, 3, 6 et 7), la fabrication de moyens (cas 4 et 8), et le contrôle-qualité (cas 2, 5 et 9) et 2 experts institutionnels (l'un membre du Gifas ${ }^{4}$ et l'autre de l'UIMM ${ }^{5}$ ). Les questions semi-

\footnotetext{
${ }^{4}$ Le Gifas (Groupement des Industries Françaises Aéronautiques et Spatiales) est une fédération professionnelle qui regroupe 366 sociétés - depuis les grands maîtres d'œuvre et systémiers jusqu'aux PMI - spécialisées dans l'étude, le développement, la réalisation, la commercialisation et la maintenance de tous programmes et matériels aéronautiques et spatiales (source : https://www.gifas.asso.fr).
} 
directives ont été ajustées au fur et à mesure des entretiens selon les réponses des répondants. D’une durée allant de 45 à 120 minutes, ils se sont déroulés de mars à juin 2016, conduits en face-à-face, enregistrés, retranscrits et validés par tous les répondants. Une clause de confidentialité a été signé entre les chercheurs et les entreprises interrogées compte tenu du caractère stratégique de leur activité : nous ne nommerons pas celles-ci et nous choisissons de les numéroter de 1 à 10 sans que cette numérotation suive un sens particulier. Tous les cas de cette étude sont adhérents à Aerospace Cluster Auvergne Rhône Alpes, association professionnelle régionale des industriels travaillant pour l'aéronautique et/ou le spatial. L'objectif est de réunir ces industriels «proches" géographiquement afin de créer des relations d'affaires et favoriser la création d'un réseau. De plus, tous les industriels interrogés sont membres de l'UIMM à l'exception du cas 6. Tous les cas sont également membres du Gifas. Notre échantillon ( $c f$. infra tableau 3) est représentatif de la population-mère, ici les sous-traitants de l'aéronautique, et est composée de PMI. Ces dernières sont considérées comme faisant partie de «l'entreprise étendue », correspondant à la quasi-intégration et aux formes traditionnelles de sous-traitance (Cagli et al., 2009). Ces preneurs d'ordres sont en principe en relation avec les donneurs d'ordres de niveau un et deux, et jamais avec l'avionneur qui se décharge ainsi de l'animation de cet ensemble de firmes.

Tableau 3 : Description de l'échantillon et des interviews

\begin{tabular}{|c|c|c|c|c|}
\hline Cas & $\begin{array}{l}\text { Principaux donneurs } \\
\text { d'ordres } \\
\end{array}$ & $\begin{array}{l}\text { Prestations pour la supply chain (SC) } \\
\text { aéronautique }\end{array}$ & $\begin{array}{c}\text { Fonction du } \\
\text { répondant }\end{array}$ & Durée \\
\hline 1 & $\begin{array}{c}\text { Airbus, Safran Aircraft } \\
\text { Engines, Safran Landing } \\
\text { Systems }\end{array}$ & $\begin{array}{l}\text { Production de tôles, lingots, billes, barres, } \\
\text { blocs, pièces matricées, forgées, poudrées } \\
\text { en acier, inox, aluminium, titane, alliage, } \\
\text { super alliage }\end{array}$ & $\begin{array}{l}\text { Responsable } \\
\text { qualité et supply } \\
\text { chain }\end{array}$ & $115^{\prime}$ \\
\hline 2 & $\begin{array}{l}\text { Airbus, Dassault, Aubert } \\
\text { \& Duval }\end{array}$ & $\begin{array}{c}\text { Prestations de service et R\&D Essais, } \\
\text { analyses et expertises sur matériaux } \\
\text { métallique et composite }\end{array}$ & PDG & $45^{\prime}$ \\
\hline 3 & Airbus & Production de pièces en aluminium & Chargé d'affaires & $117^{\prime}$ \\
\hline 4 & $\begin{array}{c}\text { Figeac Aero, } \\
\text { Mecachrome, ARM } \\
\text { Group, Nexteam Group, } \\
\text { Rossi Aero }\end{array}$ & $\begin{array}{l}\text { Outillages, réalisation sur mesure de } \\
\text { plaques ou de blocs acier, inox et alu de } \\
\text { quelques millimètres à } 25 \text { tonnes. }\end{array}$ & $\begin{array}{l}\text { Responsable } \\
\text { commercial }\end{array}$ & $47^{\prime}$ \\
\hline 5 & $\begin{array}{l}\text { Safran Electronics \& } \\
\text { Defense, Dassault, } \\
\text { Constellium, MBDA, } \\
\text { Roxel, Nexter }\end{array}$ & Contrôle non destructif & PDG & $126^{\prime}$ \\
\hline 66 & $\begin{array}{l}\text { Airbus, Boeing, } \\
\text { Embraer, Bombardier, } \\
\text { Dassault } \\
\end{array}$ & Pneu radial & $\begin{array}{c}\text { Responsable } \\
\text { communication }\end{array}$ & $58^{\prime}$ \\
\hline 7 & $\begin{array}{l}\text { Aubert \& Duval, Safran } \\
\text { Aircraft Engines, } \\
\text { Constellium, SEFIAM }\end{array}$ & $\begin{array}{l}\text { Sciage de formats suivant plans } \\
\text { Découpe de blocs titane }\end{array}$ & PDG & $82^{\prime}$ \\
\hline 8 & $\begin{array}{c}\text { Safran Electronics \& } \\
\text { Defense, Safran Aircraft } \\
\text { Engines, Safran } \\
\text { Helicopter Engines, } \\
\text { Safran Power Units, Lisi } \\
\text { Aerospace } \\
\end{array}$ & $\begin{array}{l}\text { Fabrication de machines-outils à } \\
\text { commande assistée ou numérique, } \\
\text { travaillant par enlèvement de copeaux }\end{array}$ & Directeur de site & $45^{\prime}$ \\
\hline 9 & $\begin{array}{l}\text { Aubert \& Duval, Hop } \\
\text { régional, Aéro 13, Safran }\end{array}$ & Contrôle non destructif, formations & Sales manager & $600^{\prime}$ \\
\hline 10 & Safran, Iai, Avic, Airbus, & Etudes et réalisation des lignes de & $\mathrm{CEO}$ & $60^{\prime}$ \\
\hline
\end{tabular}

${ }^{5}$ L'IUMM (Union des Industries et des Métiers de la Métallurgie) est une organisation professionnelle dont la vocation est de représenter et de promouvoir les intérêts des entreprises de la métallurgie de toutes tailles et couvrant de nombreux secteurs d'activités (source : https://uimm.fr/). 


\begin{tabular}{|c|c|c|c|c|}
\hline & Sonaca, Techspace & $\begin{array}{c}\text { production pour des modules avioniques } \\
\text { en matériaux composites. }\end{array}$ & $\begin{array}{l}\text { Responsable des } \\
\text { ventes et achats. }\end{array}$ & \\
\hline $11^{6}$ & $(\mathrm{~N} / \mathrm{A})^{*}$ & $\begin{array}{c}\text { Formations } \\
\text { Suivi des industriels réalisant une activité } \\
\text { lié à la métallurgie }\end{array}$ & $\begin{array}{c}\text { Responsable de } \\
\text { communication. }\end{array}$ & $60^{\prime}$ \\
\hline
\end{tabular}

* NA : non applicable.

\subsection{APPROCHE METHODOLOGIQUE}

L'entretien semi-directif a représenté notre principal mode de recueil des données. Nous avons tout d'abord structuré nos données grâce à une analyse thématique (Chaney, 2010). Cette méthode s'inscrit dans les prescriptions de Miles et Hubermann (1991) qui incitent aux regroupements tout en construisant des liens avec les propositions théoriques : ici, notre grille de lecture de la réalité empirique renvoie à l'école de la proximité. A l'aide du logiciel NVIVO. 11, nous avons ainsi réalisé une thématisation continue afin d'identifier les thèmes au fur et à mesure de la lecture du corpus (Paillé et Mucchielli, 2003). Un thème est considéré comme pertinent si l'on constate une récurrence dans son apparition au sein du corpus. La récurrence réside dans la similitude du sens des propos formulés. Notre objectif étant de rendre compte des représentations partagées, deux facteurs sont pris en compte pour constater l'importance des éléments des représentations : un facteur quantitatif, en choisissant de calculer les fréquences d'apparition, et un facteur qualitatif, qui revient à observer les éléments qui sont décisifs pour la signification de l'objet représenté (Moliner, 1995). D'un point de vue quantitatif, l'idée est la suivante : plus les thèmes sont acceptés par la majorité des répondants, plus le pourcentage d'apparition (ou son nombre) sera élevé. D'un point de vue qualitatif, les éléments partagés par la majorité des répondants (ou les éléments centraux) doivent être significatifs de l'objet représenté.

De notre analyse de contenu thématique, nous avons distingué le contenu des proximités (induit par le questionnaire) des effets des proximités sur la relation de sous-traitance (émergents des entretiens menés). Par ailleurs, nous avons réalisé un double codage d'un tiers des entretiens choisis au hasard (Miles et Huberman, 1991) et testé la fiabilité inter-codeurs en déterminant si les trois experts avaient codé de manière similaire et affecté les verbatims aux mêmes thèmes (Larsson, 1993). Le codage en triple aveugle nous a permis de calculer le taux de fiabilité inter-codeur, calculé selon Miles et Huberman (1991) (nombre d'accords/ [nombre total d'accords + désaccords]) : il atteint $89 \%$, ce qui est satisfaisant. Ainsi, l'écart de codage est faible et les corrélations inter-codeurs sont élevées. Nous avons choisi de nous détacher complètement du guide d'entretien dans les retranscriptions et les analyses de contenu afin de ne pas être influencé par nos croyances et nos attentes dans la recherche ainsi menée. Autrement dit, nous avons cherché à limiter les biais de confirmation (Nickerson, 1998).

\section{RESULTATS : CONTENU ET EFFETS DE LA PROXIMITE INSTITUTIONNELLE}

Suite à l'analyse de contenu thématique, il apparaît que le thème le plus récurrent dans les discours est celui des caractéristiques du secteur, duquel quatre sous-thèmes émergent comme autant de représentations partagées. Diverses attitudes comme autant d'effets de la proximité institutionnelle sur la relation de sous-traitance sont mis à jour: en particulier un effet d'enfermement (lock in) apparaît.

\subsection{CONTENU ET FREQUENCE D'APPARITION}

Nous observons dans les discours des éléments factuels et des croyances qui se confondent. Ainsi peut-on observer dans le tableau 4 les thèmes les plus récurrents qui renvoient à la

${ }^{6}$ UIMM Représentant en Auvergne, interrogé en tant qu'expert dans notre étude. 
proximité institutionnelle étudiée. Pour les acteurs interviewés, il apparaît que l'industrie aéronautique octroie une légitimité aux industriels parties prenantes. Ces derniers ont à la fois une vision positive et négative de celle-ci, tandis qu'ils considèrent ce secteur comme rigoureux.

\section{Tableau 4 : Les représentations partagées du secteur comme contenu de la proximité institutionnelle : extraits de verbatim et fréquences d'apparition}

\begin{tabular}{|c|c|}
\hline $\begin{array}{l}\text { Représentations } \\
\text { partagées }\end{array}$ & Extraits de verbatim \\
\hline \multicolumn{2}{|c|}{$48 \%(54 / 113$ occurrences $)$} \\
\hline $\begin{array}{l}\text { Secteur à forte } \\
\text { légitimité }\end{array}$ & $\begin{array}{l}\text { «Obtenir les qualifications, c'est long, c'est coûteux donc le ticket d'entrée il n'est pas } \\
\text { facilement accessible : quand on l'a, on le garde » (Cas 2). } \\
\text { "On rentre dans un monde où les règles sont partagées et les règles du jeu sont connues, } \\
\text { les principes d'excellence on sait de quoi il s‘agit et voilà » (Cas 1). } \\
\text { "Développer ce marché : oui. On souhaite partout participer » (Cas 8). } \\
\text { «L'aéronautique met effectivement cette partie normative et du coup élitiste » (Cas 7). }\end{array}$ \\
\hline \multicolumn{2}{|c|}{$31 \%(34 / 113$ occurrences) } \\
\hline $\begin{array}{l}\text { Perception } \\
\text { négative (lenteur, } \\
\text { manque } \\
\text { d'informations) }\end{array}$ & $\begin{array}{l}\text { « Oui ça change d'un avionneur à un autre... l'américain va être plus précis, ils ont une } \\
\text { personne pour chaque truc nous on est plus polyvalent et on connaîtra toute la chaine ... } \\
\text { Très lent à faire changer les mentalités » (Cas 3). } \\
\text { "Alors est-ce que c'est l'A380 ou l'A320 Néo ou l'A350 ? Je pense qu'on a beaucoup } \\
\text { travaillé pour l'A350 à une certaine époque, on a dû travailler aussi pour l'A320 Néo, } \\
\text { l'A380 c'est évident, l'A400M certainement, mais on n'a pas assez de visibilité » (Cas } \\
\text { 2). } \\
\text { "Nous savons sur quel programme on travaille au pire on le devine, peu importe. } \\
\text { Comment on motive un salarié ? Tu lui dis tu es en train de travailler sur une pièce de } \\
\text { l'A380. Il faut être fier de ce qu'on fait » (...) « Si vous voulez, ce n'est pas écrit de } \\
\text { manière noir sur blanc sur les commandes que nous avons » (Cas 7). } \\
\text { "En théorie, on devrait le savoir, on devrait connaitre la finalité. Je dirai que dans } 80 \% \\
\text { des cas on ne sait pas sur quoi on travaille» (Cas 4). }\end{array}$ \\
\hline $\begin{array}{l}\text { Perception } \\
\text { positive }\end{array}$ & $\begin{array}{l}\text { «C'est quand même toujours très valorisant de travailler pour des technologies de } \\
\text { pointe comme l'aéronautique, parce que les opérateurs le perçoivent comme ça » (Cas } \\
\text { 2). } \\
\text { «L'aéronautique c'est le top, le prestige » (Cas } 3) \text {. } \\
\text { « Nous, le développement aéronautique, ça fait partie de nos priorités (...). Je crois que } \\
\text { c'est l'objectif de tous » (Cas } 4)\end{array}$ \\
\hline \multicolumn{2}{|c|}{$21 \%(23 / 113$ occurrences $)$} \\
\hline Secteur rigoureux & $\begin{array}{l}\text { «Donc tout ça est écrit, et l'opérateur doit lire et respecter scrupuleusement le mode } \\
\text { opératoire pour effectuer les essais pour lequel il est qualifié » (Cas } 2) \text {. } \\
\text { «Il nous donne des plans ce sont des pièces normées, connues. Tout est figé » (Cas 3). } \\
\text { «La culture de la conformité et de la reproductivité c'est que toute pièce doit être } \\
\text { conforme aux pièces d'essai » (Cas 1). } \\
\text { « Le poids normatif dans l'aéronautique est énorme » (Cas 5). }\end{array}$ \\
\hline
\end{tabular}

En résumé, la proximité institutionnelle se traduit par une convergence des représentations concernant les cahiers des charges, certifications et les relations donneurs d'ordresfournisseurs. Alors que les comportements requièrent l'observation des actions effectives par les acteurs, les attitudes en tant que jugement évaluatif sont accessibles par le discours. C'est ainsi que les acteurs mettent en avant la légitimité et la rigueur du secteur. Simultanément des perceptions positives et négatives se révèlent dans les discours se traduisant par autant d'attitudes que nous avons pu analyser et discuter dans ce travail.

\subsection{ATTITUDES ET FREQUENCE D'APPARITION}

Le tableau 5 révèle les éléments émergents de nos entretiens, comme autant d'attitudes communes. Il faut noter une tendance chez nos interlocuteurs à conserver en interne une capacité d'expertise, de production et de contrôle la plus élevée possible. De même, il est 
parfois nécessaire d'expliciter aux salariés concernés les exigences des clients. Simultanément, ils reconnaissent l'existence de processus d'apprentissage mutuel entre partenaires.

Tableau 5 : Les attitudes comme effets de la proximité institutionnelle : extraits de verbatim et fréquences d'apparition

\begin{tabular}{|c|c|}
\hline Attitudes & Extraits de verbatim \\
\hline \multicolumn{2}{|c|}{$38 \%(42 / 111$ occurrences $)$} \\
\hline Internaliser & $\begin{array}{l}\text { «Pour moins être moins sensible aux sous-traitants on doit tout faire... » (Cas 5). } \\
\text { «(...) tout est fait chez nous. Ça, on est complètement autonome là-dessus, pour des raisons } \\
\text { de confidentialité » (Cas 6). } \\
\text { «On se doit d'avoir, nous, l'expertise et la maitrise de ce qu'on fait donc on ne sous-traite } \\
\text { quasiment pas. Si on sous-traite l'usinage d'éprouvette par exemple. Mais dans les essais on } \\
\text { ne sous-traite pas » (Cas } 2) \text {. } \\
\text { «Plus le produit est travaillé chez nous plus la matière reste chez nous. Donc on peut } \\
\text { reprendre ces chutes et faire de l'économie circulaire » (Cas 3). }\end{array}$ \\
\hline Traduire & « On s’organise pour transcrire les exigences de nos clients » (Cas 1). \\
\hline Contrôler & $\begin{array}{l}\text { «Il faut adapter le contrôle, donc en interne on a écrit pour chaque méthode des règles pour } \\
\text { appliquer les contrôles quand il n’y a rien... » (Cas 5). } \\
\text { " Nous, généralement, on est assez exigeants vis-à-vis de nos usines parce qu'en fait, un } \\
\text { audit Boeing va être différent d'un audit Airbus, donc nous généralement, on se met une } \\
\text { strate au-dessus comme ça on est sûr de tout passer. On est assez exigeants » (Cas 6). }\end{array}$ \\
\hline \multicolumn{2}{|c|}{$18 \%(20 / 111$ occurrences $)$} \\
\hline $\begin{array}{l}\text { Apprentissage } \\
\text { mutuel }\end{array}$ & $\begin{array}{l}\text { "La culture aéronautique nous permet de nous faire monter en compétences et surtout } \\
\text { d'élargir un peu notre panel de connaissance au niveau de nos partenaires » (Cas 2). }\end{array}$ \\
\hline
\end{tabular}

Nos répondants déclarent construire des mécanismes de coordination intra-entreprise et interentreprises permettant de faire barrière à une proximité institutionnelle forte. En premier lieu, nous observons une volonté d'internaliser le maximum d'opérations pour préserver la confidentialité de celles-ci et pour développer la maitrise de leurs savoir-faire. En second lieu, nous notons des actions de traduction et de contrôle permettant de répondre aux cahiers des charges parfois mal aisés à déchiffrer, aux exigences de rigueur ou encore au manque d'informations.

\section{DISCUSSION}

Nos résultats sont issus d'une analyse de contenu thématique. Elle nous autorise à commenter à la fois ce qui constitue la proximité institutionnelle et les effets de cette dernière qui lie les industriels et experts aéronautiques auvergnats interrogés.

\subsection{CONTENU DE LA PROXIMITE INSTITUTIONNELLE : DES REPRESENTATIONS PARTAGEES POSITIVES ET NEGATIVES}

Sur le plan du contenu de la proximité institutionnelle, nos résultats sont à la fois confirmatoires et novateurs. Confirmatoire dans le sens où l'industrie aéronautique est perçue par le grand public et présentée par les grands industriels de façon très positive. Nos résultats montrent que les industriels et experts auvergnats questionnés partagent cette représentation positive de l'aéronautique (avenir positif, secteur valorisant, légitimité, vitrine technologique, univers technique) confirmant de nombreux travaux antérieurs (Frenken, 2000 ; Mazaud, 2006 ; Cagli et al., 2009 ; Jalabert et Zuliani, 2009). Ainsi, ce secteur provoque des retombées bénéfiques à l'ensemble de l'industrie nationale et reste un créateur d'emploi industriel en France. Il ressort par ailleurs clairement de notre échantillon que l'industrie aéronautique reste une vitrine technologique qui octroie une forte légitimité aux industriels qui y sont présents en termes de capacité à respecter des contraintes de coûts, de délais et de qualité (Frigant et Talbot, 2005). Cette légitimité, qui se concrétise par des certifications, peut être mobilisée 
dans d'autres domaines industriels. Ainsi, selon la perception des répondants, les exigences de certification sont perçues positivement parce qu'elles cautionnent les industriels. Au final, travailler pour l'industrie aéronautique constitue un avantage concurrentiel que l'on peut déployer dans d'autres secteurs. La proximité institutionnelle est donc perçue comme un vecteur de confiance institutionnelle (Zucker, 1986) en étant source d'affaires et en mettant en place des barrières à l'entrée par la mise en place des règles du jeu (Williamson, 1993), des certifications (Noteboom, 2000) et des représentations qui en découlent (Hofstede, 1993 ; Scott, 1995).

De façon novatrice cette fois, notre travail révèle que la perception collective des soustraitants auvergnats est parfois plus critique (secteur lourd, contraignant, difficile, absence d'interlocuteur, lenteur du marché) que l'image traditionnellement renvoyée par les leaders du secteur. Ainsi, les exigences de certification apparaissent aux yeux de certains répondants très contraignantes et difficiles à comprendre en particulier pour les petites structures. L'aspect contraignant peut s'expliquer par la nécessité de garantir un très haut niveau de sécurité dans le transport aérien, ce qui conduit à des exigences de certification très fortes. La lenteur du marché renvoie à la durée moyenne d'un programme aéronautique qui s'exprime en dizaine d'années (quarante ans pour un programme qui rencontre un fort succès commercial comme le B747) et des délais de retour sur investissements qui peuvent atteindre plus de dix ans (cas de l'A320).

Plus surprenante est l'idée que les interlocuteurs des sous-traitants sont jugés absents, dans une industrie qui a développé une chaîne de valeur très complexe et très intégrée. Il apparait que la circulation d'informations entre donneurs et preneurs d'ordres n'est pas parfaite, ces derniers constatant un manque d'informations sur la nature des programmes aéronautiques concernés par la délégation des tâches. Si les relations de sous-traitance apparaissent comme très intenses entre les avionneurs et leurs fournisseurs de rang 1 et 2, elles semblent s'atténuer lorsqu'on interroge des acteurs de rang 3 ou 4. Ainsi, une communication non effective et un manque d'informations rendent difficiles la réalisation des tâches demandées dans le cadre de la relation de sous-traitance.

\subsection{EFFETS DE LA PROXIMITE INSTITUTIONNELLE}

Dans le discours des répondants, nous repérons les effets de la proximité institutionnelle sur les relations productives entre industriels. Ces éléments sont alors émergents et non induits par le guide d'entretien. Nous observons que les effets de la proximité institutionnelle pèsent sur ces industriels et orientent leurs pratiques. Dans la première catégorie d'effets, les modèles de pensées et de faire sont similaires (Hodgson, 2002) ; dans l'autre catégorie, les rôles sont distribués pour assurer la coordination (Kirat et Lung, 1999). En outre, apparaît un effet inattendu d'enfermement (lock in) institutionnel.

\subsubsection{Similarité et complémentarité des attitudes}

Côté similarité des attitudes, nous avons observé que nos répondants adoptent une stratégie identique de défense de leurs savoir-faire en termes de production et de contrôle qualité. Ce maintien leur permet de conserver une place dans la chaîne de valeur aéronautique qui se caractérise par une concurrence accrue entre preneurs d'ordres. Les principaux donneurs d'ordres n'hésitent pas pour des raisons de coût à faire appel à des PME par exemple localisées au Maghreb ou en Asie. Les PME auvergnates, ne pouvant rivaliser sur les coûts, ont alors intérêt à maintenir des compétences spécifiques leur permettant de rester des soustraitants de spécialité. De fait, ils appartiennent à un même segment stratégique (en l'occurrence le travail des métaux, $c f$. tableau 3) et sont positionnés dans les rangs les plus inférieurs de la chaine de valeur aéronautique. Cette position les contraint à accepter les règles du jeu de l'industrie aéronautique, comme le démontre le respect dont ils font preuve des 
normes imposées par les principaux donneurs d'ordres. Ajoutons que la similarité des attitudes observées est encore renforcée par la nature même de notre échantillon. En effet, plusieurs éléments expliquent la congruence des discours des répondants: les acteurs interrogés occupent des fonctions d'encadrement et sont en relation avec les clients et/ou les fournisseurs. Ils partagent la même culture nationale et régionale, tandis qu'ils interagissent au sein des mêmes associations professionnelles dont ils partagent les règles et les valeurs.

Côté complémentarité des attitudes, chacun tient son rôle pour répondre aux attentes très normées du donneur d'ordres. Il s'agit là d'une caractéristique d'un secteur attentif aux questions de sécurité et donc de qualité des produits. En effet, la segmentation de la supply chain configure une organisation des relations inter-entreprises assise sur une division cognitive du travail elle-même basée sur une hiérarchie des compétences et sur la décomposition du produit. Pour l'ensemble des sous-traitants composant notre échantillon, la fabrication des composants requiert des connaissances "limitées » aux caractéristiques des composants concernés (Cagli et al., 2009).

\subsubsection{Effet d'enfermement}

Ce travail montre en outre que la proximité institutionnelle peut générer un effet d'enfermement (lock in) (Grabher, 1993). Nos entretiens révèlent que les normes contenues dans les cahiers des charges sont parfois difficilement compréhensibles pour nos interlocuteurs. L'incompréhension réside soit dans le langage utilisé (un langage managérial et pas le «jargon» technique de coutume dans le métier) ou soit dans le manque d'informations vis-à-vis du produit à fournir, ce qui pose un problème en termes d'implication. Les sous-traitants mettent alors en place des pratiques de traduction pour réaliser les commandes, pour tenter de motiver les techniciens et pour ajouter de la valeur à leurs tâches. Il s'agit alors de limiter l'inertie des collaborateurs très contraints par le respect des normes imposées par les donneurs d'ordres. La perception négative du secteur aéronautique exprimée par les acteurs alimente et est alimenté par cet effet d'enfermement. Le champ des possibles est effectivement réduit : plus les acteurs sont au bas de la chaine plus leur pouvoir d'intervention en cas de probléme (par exemple dans le cas d'une simplification des procédés possible) est mis à mal même, s'ils ont les solutions en interne. La proximité institutionnelle réduit ici les possibilités d'actions des acteurs et ne favorise pas des attitudes en faveur par exemple du transfert de connaissances. Le cadre institutionnel mis en avant favorise un encastrement culturel que l'on retrouve d'ailleurs dans les supply chains global en général (Wu et Pullman, 2015). Le niveau contraignant est dans ce cas macro-institutionnel prescrivant ainsi à la fois les comportements et les stratégies. Il n'y a pas de contre-effets, sinon de façon isolée ( $c f$. les actions de traductions du cas 5 ou du cas 7 en interne) : ces micros actions ne permettent pas de modifier les logiques institutionnelles.

Par ailleurs, il existe une difficulté à accéder aux informations sectorielles, d'autant que suite à la fusion des deux régions Auvergne et Rhône Alpes, l'association professionnelle des acteurs de l'aéronautique auvergnate AVIA a fusionné avec Aerospace Cluster Auvergne Rhône-Alpes, générant une plus grande difficulté pour les acteurs auvergnats d'accès à l'information. En outre, les PMEs composant notre échantillon se sentent en danger tant les exigences de leurs donneurs d'ordres sont élevées et contraignent leurs actions. Les soustraitants de rang 3 et 4 se disent empêchés de monter dans la chaine de valeur en proposant plus que ce qu'il leur ait demandé dans le cahier des charges grâce à leurs connaissances et leurs pratiques issues des autres activités dans lesquelles ils évoluent.

Dès lors, plus on descend dans la chaine de valeur, plus les institutions partagées deviennent problématique aux yeux des industriels concernés tant le manque de communication, de transparence et la distribution du pouvoir limitent les possibilités d'action des sous-traitants. Le risque qu'une proximité institutionnelle forte devienne défavorable au développement de 
nouvelles idées et aux innovations (effet d'enfermement) en créant une inertie institutionnelle (Boschma, 2005) est dans notre cas réel.

\section{CONCLUSION}

Finalement, après avoir constaté que peu de travaux s'attachaient à analyser le contenu et les effets de la proximité institutionnelle, nous avons défini cette dernière. Cet article propose ensuite une méthode pour analyser des représentations partagées qui constituent cette proximité institutionnelle grâce aux discours des répondants. A partir de l'analyse thématique et des fréquences d'apparition, nous donnons une preuve empirique de l'existence de cette proximité que partagent les sous-traitants de l'aéronautique localisés en Auvergne. Il apparaît notamment que ces derniers ont une perception certes positive du secteur mais aussi négative, ce dernier point étant un résultat novateur pour une industrie traditionnellement mis en avant. Un effet d'enfermement est constaté, freinant les prises d'initiative et limitant la capacité d'innovation des sous-traitants de rang inférieur. La gestion de la proximité institutionnelle par les acteurs passe alors par des stratégies de contournement : l'internalisation, la traduction, le contrôle et l'apprentissage mutuel sont autant de solutions opérationnelles permettant de s'affranchir du poids institutionnel de l'industrie aéronautique.

Ce travail de nature exploratoire n'est pas exempt de limites et nécessite des investigations supplémentaires. Nous nous sommes limités à une observation de la proximité institutionnelle sous l'angle informel : l'étude de la dimension formelle supposerait un accès aux contrats de sous-traitance qui reste inaccessibles aux chercheurs pour des raisons de confidentialité. De plus, en nous intéressant à la perception de la réalité de chacun de nos managers ou experts interrogés dans notre étude et en décrivant la proximité institutionnelle à partir de leurs propos, nous nous sommes rattachés à une position qui accorde une grande importance aux connaissances contextuelles des répondants (Tsoukas, 1989), adaptés à l'étude d'un secteur mais ne permettant pas de généraliser les résultats. Nous n'avons par ailleurs pas observé directement les comportements qui traduisent concrètement les actions.

Au-delà de ces limites, cet article permet d'envisager deux perspectives de recherche. En premier lieu, il est possible de compléter notre analyse de données par des méthodes issues des sciences cognitives à l'instar d'une analyse prototypique (Vergès, 1995) ou d'une analyse selon la technique de mise en cause afin d'identifier les éléments centraux des représentations et réaliser une carte cognitive. Ainsi, en second lieu, il serait pertinent de réaliser une étude confirmatoire auprès d'un échantillon plus important. En effet, il serait opportun de tester au sein d'un modèle conceptuel l'impact de la proximité institutionnelle sur la nature des relations de sous-traitance des acteurs interrogés. Nous pourrions alors confirmer l'impact négatif de la proximité institutionnelle sur les relations des sous-traitants de rang inférieur.

\section{BIBLIOGRAPHIE}

ABRIC J.-C. (1994), Pratiques sociales et représentations, Paris, Presses Universitaires de France.

ADAM-LEDUNOIS S. A., BAUDASSE T., RENAULT S. (2010), «Proximité et capital social : le cas des Parcs Industriels Fournisseurs », Management \& Avenir, N³, p. 14-34.

ADAM-LEDUNOIS S., RENAULT S. (2008). «La coordination spatiale des Parcs industriels fournisseurs », Revue française de gestion, Vol. 4, N¹84, p. 167-180.

AMABLE B., PALOMBARINI S. (2005), «L'économie politique n'est pas une science morale » Lectures, Publications reçues (2005).

ANGUÉ K., MAYRHOFER U. (2010), « Coopérations internationales en R\&D : les effets de la distance sur le choix des pays partenaires », M@n@gement, Vol. 13, N¹, p. 1-37.

AOKI M. (2006), Fondements d'une analyse institutionnelle comparée, Albin Michel. 
ARTHUR W.B. (1989), " Competing technologies, increasing returns, and lock-in by historical events ». The Economic Journal, 99 (March), p. 116-131.

BALLAND P. A. (2012), "Proximity and the evolution of collaboration networks: evidence from research and development projects within the global navigation satellite system (GNSS) industry », Regional Studies, Vol. 46, №6, p. 741-756.

BECUE M., BELIN J., TALBOT D. (2014), «Relational rent and underperformance of hub firms in the aeronautics value chain »,M@n@gement, Vol.17, º2, p. 110-135.

BELlet M., COLlETIS G., LUNG Y. (1993), «Economie de proximités », Revue d'Economie Régionale et Urbaine, N³, Numéro Spécial.

BENNERA M., WALDFOGELB J. (2008), "Close to you ? Bias and precision in patentbased measures of technological proximity », Research Policy, Vol. 37, N9, p. 1556-1567.

BERGER P., LUCKMANN T. (1966). The Social Construction of Reality, New York Anchor.

BOSCHMA R. (2005), " Proximity and Innovation: A Critical Assessment », Regional Studies, Vol. 39, $\mathrm{N}^{\circ} 1$, p. 61-74.

BOSCHMA R., FRENKEN K. (2009), "Some notes on institutions in evolutionary economic geography », Journal of Economic Geography, Vol. 85, №2, p. 151-158.

BOUBA-OLGA O., GROSSETTI M. (2008), "Socio-économie de proximité », Revue d'Economie Régionale et Urbaine, №3, p. 311-328.

BRESCHI S., LISSONI F., MALERBA F. (2003), "Knowledge-relatedness in firm technological diversification ", Research Policy, Vol. 32, №1, p. 69-87.

BROEKEL T., BOSCHMA R. (2012), « Knowledge networks in the Dutch aviation industry: The proximity paradox», Journal of Economic Geography, Vol. 12, ํ2, p. 409-433.

CAGLI A., KECHIDI M., LEVY R. (2009), « Gestion stratégique de la supply chain et firme pivot dans le secteur aéronautique », Revue Française de Gestion Industrielle, Vol. 28, №2, p. 124-139.

CARRINCAZEAUX C., GROSSETTI M., TALBOT D. (eds), (2008), « Clusters, proximities and networks », Special Issue of European Planning Studies, Vol.16, N5, p. 613-621.

CARTON S., FARASTIER A. (2012), « Intégration des connaissances client dans un projet en systèmes d'information : influence de l'environnement de connaissance du projet», Systèmes d'information \& management, Vol. 17, N², p. 39-80.

CHANEY D. (2010), "L'apport des cartes cognitives à l'analyse des représentations mentales », Recherche et Applications en Marketing, Vol. 25, N², p. 93-115.

COLLETIS G. (2010), "Co-évolution des territoires et de la technologie : une perspective institutionnaliste », Revue d'Économie Régionale \& Urbaine, N², p. 235-249.

DAVID A. (2003), "Etude de cas et généralisation scientifique en sciences de gestion », Revue Sciences de Gestion, № 39.

DESCOMBES V. (1996), Les institutions du sens, Les éditions de minuit, Paris.

DETCHENIQUE G., LOILIER T. (2016), «La régénération stratégique d'un réseau inter organisationnel territorialisé », Revue Française de Gestion, Nㅜ, p. 11-31.

DIMAGGIO P.J., POWELL W.W. (1983), «The iron cage revisited: institutional isomorphism and collective rationality in organizational fields », American Sociological Review, Vol. 48, N², p. 147-160.

DUPUY C., TORRE A. (2004), Confiance et proximité, In Pecqueur B. et Zimmermann J.B, Economie de Proximités, Hermès, Paris, p. 65-87.

DURKHEIM E. (1898), "Représentations individuelles et représentations collectives », Revue de Métaphysique et de Morale, №6, p. 273-302.

EHLINGER S. (1998), «Les représentations partagées au sein des organisations : entre mythe et réalité », Association Internationale en Management Stratégique, In 7th Conférence Internationale de l'AIMS, Mai 2017, Louvain-la-Neuve. 
EMIN S., SAGOT-DUVAUROUX D. (2016), «L'émergence de dynamiques coopératives: l'exemple d'un réseau d'entreprises créatives co-localisées. Une approche par l'économie des proximités ». Géographie Economie Société, Vol. 18, N4, p. 525-550.

FISS P. (2007), "A Set-Theoretic Approach to Organizational Configurations ", The Academy of Management Review, Vol. 32, N4, p. 1180-1198.

FRENKEN K. (2000), «A complexity approach to innovation networks: The case of the aircraft industry (1909-1997) », Research Policy, Vol. 29, N², p. 257-272.

FRIGANT V., TALBOT D. (2005), «Technological determinism and modularity: lessons from a comparison between Aircraft and Auto Industries in Europe ", Industry and Innovation, Vol. 12, N³, p. 337-335.

GARDES N., BEGUINET S., LIQUET J. (2013), « Du concept d'image à sa mesure, une approche par les représentations sociales : Application au secteur bancaire français », Revue Des Sciences De Gestion, №3, p. 157-166.

GEELS F. W. (2014), « Reconceptualising the co-evolution of firms-in-industries and their environments: Developing an inter-disciplinary Triple Embeddedness Framework », Research Policy, Vol. 43, N², p. 261-277.

GILLY J. P., LUNG Y. (2005), "Proximités, secteurs et territoires », Groupement de Recherches Economiques et Sociales (GRES).

GILLY J. P., TORRE A. (2000), Dynamiques de proximité, L'Harmattan, Paris.

GOMEZ P.Y., ROUSSEAU A., VANDANGEON-DERUMEZ I. (2011), " Distance et proximité : esquisse d'une problématique pour les organisations ", Revue Française de Gestion, Nº 4, p. 13-24.

GRABHER G. (1993), The embedded firm. On the socio-economics of industrial networks, Routledge, London.

HAMADI Z. B., CHAPELLIER P., VILLESEQUE-DUBUS F. (2014), «Innovations budgétaires en PMI : l'influence du secteur d'activité et du profil du dirigeant », Innovations, $\mathrm{N}^{\circ} 1$, p. 223-252.

HAUTALA J. (2011), "Cognitive proximity in international research groups », Journal of Knowledge Management, Vol. 15, N4, p. 601-624.

HISLOP D. (2000), «Environmental constraints and sectoral recipes: strategy change in Britain's military industrial base », Journal of Management Studies, Vol. 37, N5, p. 687-703. HODGSON G. (2002), « Reclaiming habit for institutional economics », Journal of Economic Psychology, Vol. 25, N5, p. 651-660.

HODGSON G. (2006), «What are institutions? », Journal of Economic Issues, Vol. 40, №1, p. 1-25.

HOFSTEDE G. (1993), "Cultural constraints in management theories », Academy of Management Perspectives, Vol. 7, $\mathrm{N}^{\circ} 1$, p. 81-94.

JALABERT G., ZULIANI J.M. (2009), Toulouse, l'avion et la ville, Privat, Toulouse.

JODELET D. (2003), «Représentations sociales : un domaine en expansion », Sociologie d'aujourd'hui, $\mathrm{N}^{\circ} 7$, p. 45-78.

KECHIDI M., TALBOT D. (2010), «Institutions and coordination: what is the contribution of a proximity-based analysis? The case of Airbus and its relations with the subcontracting network », International Journal of Technology Management, Vol. 50, №3/4, p. 285-299.

KIRAT T., LUNG Y. (1995), Innovations et proximités : le territoire lieu de déploiement des processus d'apprentissage, In Lazaric N., Monnier J.M., Coordination économique et apprentissage des firmes, Economica, Paris, pp. 206-227.

KIRAT T., LUNG Y. (1999), «Innovation and proximity. Territories as loci of collective learning processes », European Urban and Regional Studies, Vol. 6, Nº1, p. 27-38. 
KLIMAS P. (2016), «Organizational culture and coopetition : An exploratory study of the features, models and role in the Polish Aviation Industry ", Industrial Marketing Management, Vol. 53, p. 91-102.

KLIMAS P., TWAROG S. (2015), « Inter-organizational proximity in the context of logistics research challenges », LogForum, Vol. 11, N¹, p. 109-117.

KNOBEN J., OERLEMANS L.A.G (2006), «Proximity and inter-organizational collaboration : A literature review », International Journal of Management Reviews, 8(2), p. 71-89.

LAGROYE J., FRANÇOIS B., SAWICKI F. (2006), Sociologie Politique, 5e édition, Presses de Sciences Po et Dalloz, Paris.

LAROCHE H. (1995), «From Decision to Action in Organizations : Decision-making as a Social Representation », Organization Science, Vol. 6, N²1, p. 62-75.

LARSSON R. (1993), " Case survey methodology: Quantitative analysis of patterns across case studies », Academy of Management Journal, Vol. 36, N6, p. 1515-1546.

LAURIOL J., PERRET V., TANNERY F. (2008), "Stratégies, espaces et territoires. Une introduction sous le prisme géographique », Revue française de gestion, Vol. 4, $\mathrm{N}^{\circ} 184$, p. 91103.

LAZEGA E. (1994), Analyse de réseaux et sociologie des organisations », Revue Française de Sociologie, Vol. 35, $\mathrm{N}^{\circ}$ 2, p. 293-320.

LEROUX I., PUJOL L., RIGAMONTI E. (2012), "La gouvernance du microcrédit professionnel entre jeux de proximité et lock-in effect » in Gaillard R., Argent des particuliers médiations sociales, Rapport pour le Conseil Régional des Pays de la Loire, décembre.

LOILIER T. (2010), «Innovation et territoire : Le rôle de la proximité géographique ne doit pas être surestimé », Revue française de gestion, Vol. 36, $\mathrm{N}^{\circ} 1$, p. 15-35.

LU V.N., PLEWA C., HO J. (2016), « Managing governmental business relationships: The impact of organisational culture difference and compatibility», Australasian Marketing Journal, Vol. 24, p. 93-100.

MARROCU E., PACI R., USAI, S. (2013), «Proximity, networking and knowledge production in Europe : What lessons for innovation policy ?», Technological Forecasting And Social Change, Vol. 80, No8, p. 1484-1498.

MARTINEZ-FERRERO J., GARCIA-SANCHEZ I. M. (2016), « Coercive, normative and mimetic isomorphism as determinants of the voluntary assurance of sustainability reports ", International Business Review, Vol. 26, $\mathrm{N}^{\circ} 1$, p. 1-17.

MAZAUD F. (2006), « De la firme sous-traitante de premier rang à la firme-pivot. Une mutation de l'organisation du système productif », Revue d'Economie Industrielle, Vol. 113, $\mathrm{N}^{\circ} 1$, p. 45-60.

MICHEL G. (1999), «L'évolution des marques : approche par la théorie du noyau central », Recherche et Applications en Marketing, Vol. 14, N4, p. 32-53.

MILES M. B., HUBERMAN A. M. (1991), Analyse des données qualitatives : recueil de nouvelles méthodes, De Boeck et Larcier.

MOATI P. (2013), «Représentations et dynamique sectorielle. Cadre d'analyse et application au secteur de la distribution », Revue d'Economie Industrielle, Vol. 142, №2, p. 147-186.

MOLINA-MORALES F. X., BELSO-MARTINEZ J. A., MAS-VERDU F., MARTINEZCHAFER L. (2015), «Formation and dissolution of inter-firm linkages in lengthy and stable networks in clusters », Journal of Business Research, Vol. 68, ํ7, p. 1557-1562.

MOLINER P. (1995), "A two-dimensional model of social representations ", European Journal of Social Psychology, Vol. 25, N¹, p. 27-40.

MOSCOVICI S. (1976), La psychanalyse, son image et son public, Paris, PUF.

NEGURA L. (2006), "L'analyse de contenu dans l'étude des représentations sociales ». Sociologies, Théories et recherches. 
NICKERSON R. S. (1998), «Confirmation bias: A ubiquitous phenomenon in many guises », Review of General Psychology, N², p. 175-220.

NOTEBOOM, B. (2000), Learning and Innovation in Organizations and Economies, Oxford University Press, Oxford.

NORTH D. (1994), «Economic Performance Through Time », American Economic Review, Vol. 84, N³, p. 359-368.

PAILLE P., MUCCHIELLI A. (2003), L'analyse qualitative en sciences humaines et sociales, Paris, Armand Colin.

PECQUEUR B., ZIMMERMANN J.B. (2004), Economie de Proximités, Paris, Hermès, Lavoisier.

PHANUEL D., RENAULT S. (2015), « Le partage de vision des acteurs : un enjeu majeur du management de projet », Management \& Avenir, Vol. 75, N¹, p. 129-145.

PHILLIPS M. E. (1994), "Industry mindsets: Exploring the cultures of two macroorganizational settings », Organization Science, Vol. 5, N³, p. 384-402.

PIHEL L., JOURNE B. (2016), "La fabrique du territoire : travail aux frontières ", Management \& Avenir, Vol. 83, N¹, p. 115-119.

PONDS R., VAN OORT F., FRENKEN K. (2007), « The geographical and institutional proximity of research collaboration », Regional Science, Vol. 86, N³, p. 423-443.

RALLET A. (2002), "L'économie de proximités », Etudes et Recherches sur les Systèmes Agraires et le Développement, p. 11-25.

ROJOT J. (2005), Théorie des Organisations, Deuxième édition, Editions Eska, Paris.

RYCHEN F., ZIMMERMANN J.B. (2008), "Clusters in the Global Knowledge-based Economy: Knowledge Gatekeepers and Temporary Proximity », Special Issue of Regional Studies, Vol. 42, $\mathrm{N}^{\circ} 6$, p. 767-776.

SCHMITZ T., SCHWEIGER B., DAFT J. (2016). « The emergence of dependence and lockin effects in buyer-supplier relationships-A buyer perspective », Industrial Marketing Management, Vol. 55, p. 22-34.

SCOTT W.R. (1995), Institutions and Organizations, Sage, Thousand Oakes, CA.

SHAW L. (2015), «Mechanics and dynamics of social construction: Modeling the emergence of culture from individual mental representation », Poetics, Vol. 52, p. 75-90.

SUH T., HOUSTON M. B. (2010). «Distinguishing supplier reputation from trust in buyersupplier relationships ». Industrial marketing management, Vol. 39, N5, p. 744-751.

TALBOT D. (2013), «Clustérisation et délocalisation : les proximités construites par Thales Avionics », Revue Française de Gestion, Vol. 39, №234, p.15-26.

TALBOT D. (2008), «Les institutions créatrices de proximités », Revue d'Economie Régionale et Urbaine, $\mathrm{N}^{\circ} 3$, p. 289-310.

TORRE A. (2014), «Relations de proximité et comportements d'innovation des entreprises des clusters », Revue française de gestion, N5, p. 49-80.

TORRE A., RALLET A. (2005), «Proximity and localization », Regional Studies, Vol. 39, $\mathrm{N}^{\circ} 1$, p. 47- 60 .

TSOUKAS H. (1989), "The validity of ideograph research explanations », Academy of Management Review, Vol. 14, N²42, p. 551-561.

VERGÈS P. (1995), «Représentations sociales partagées, périphériques, indifférentes, d'une minorité : méthodes d'approche », Les Cahiers internationaux de psychologie sociale, Vol. 28, p. 77-95.

WILLIAMSON O.E. (1993), " Transactions costs Economics Organization Theory », Industrial and Corporate Change, Vol. 2, $\mathrm{N}^{\circ} 2$, p. 107-156.

WU Z., PULLMAN M. E. (2015), « Cultural embeddedness in supply networks », Journal of Operations Management, Vol. 37, p. 45-58.

YIN R. (1990), Case Study Research - Design and methods, Sage. 
ZUCKER L.G. (1986), " Production of trust : institutional sources of economic structure, 1840-1920 ", Research in Organizational Behavior, (sous la dir. de) B.M. Staw et L.L. Cummings, Vol. 8, p. 53-111. 